A Abbasoglu, A Ecevit, B Ozdemir, D Anuk Ince, A Kurt, A Tarcan. Pediatrics, Baskent University Faculty of Medicine, Ankara, Turkey

Background and Aims To determine the demography, clinical manifestations and the most common organism isolated of the urinary tract infection (UTI) in the newborn infants who were admitted to the neonatal intensive care unite (NICU).

Methods Newborn infants diagnosed with UTI were investigated retrospectively, clinical and demographic characteristics of infants were collected from the medical records in the NICU. Urine cultures were obtained by suprapubic aspiration or urinary cathater.

Results Fifty-one infants were included in this study. The mean $( \pm S D)$ gestational age and weight of infants were $31.53 \pm 4.32$ weeks, and $1724.90 \pm 902.21 \mathrm{~g}$ respectively. Male patients accounted for $\% 56.9$ of the study group. Infants born with cesarian section were $\%$ 86.3. The median age for the urine culture was $31.53 \pm 4$.3days. Klebsiella pneumania was the dominant microorganism isolated in 22 patients (43.13\%), followed by Escherichia coli in 13 patients (25.4\%). The most common presenting symptoms were vomitting in $39(76.5 \%)$ infants, desaturation in $34(66.7 \%)$ infants, tachycardia in $31(60.8 \%)$ infants, apnea in $21(41.2 \%)$ infants and jaundice in $18(35.3 \%)$ infants.

Conclusions The incidence of UTI in newborn infants is $0.1-1 \%$ and it can be as high as $10 \%$ in low-birthweight and preterm babies. The presentation of UTI in the neonatal infants is non-specific and the most common clinical manifestations are vomitting, fever, enteral feeding intolerance, apnea and bradycardia. In this study, desaturation and tachycardia are also shown as presenting manifestations of UTI. Klebsiella pneumania was the dominant microorganism isolated in $22(43.13 \%)$ patients in our study.

\section{EFFECTS OF INTRAPARTUM ANTIBIOTIC PROPHYLAXIS ON NEWBORN MICROBIOTA}

doi:10.1136/archdischild-2012-302724.1334

'L Corvaglia, 'E Legnani, ${ }^{2} \mathrm{D}$ Di Gioia, ${ }^{3} \mid$ Aloisio, ' $\mathrm{S}$ Martini, ' $\mathrm{M}$ Oss, ${ }^{2} \mathrm{~B}$ Biavati, ${ }^{\prime} \mathrm{G}$ Faldella. 'Neonatology and Intensive Care Unit - Sant'Orsola-Malpighi Hospital - University of Bologna; 'Departement of Agroenviromental Science and Technologie, University of Bologna; ${ }^{3}$ Departement of Agroenviromental Science and Technologie - University of Bologna, Bologna, Italy

Background and Aims Group B Streptococcus (GBS) early-onset bacterial sepsis is an important cause of neonatal morbidity and mortality. In the last decade, after the introduction of intrapartum antibiotic prophylaxis in pregnant women during labor and delivery, the sepsis-associated death rates have declined. The aim of this study is to evaluate the effects of antibiotic treatment of pregnant women GBS-positive on early colonization of bacteria in the newborn gut, which is known to be related to immunity development. Methods Thirty-four vaginal delivered and breastfed newborns were enrolled; 17 had mothers GBS-positive treated with $2 \mathrm{~g}$ of Ampicillin and 17 had mothers GBS-negative (control group).

Two-hundred milligrams faeces were collected for each subject and processed for DNA extraction, performed with OIAamp DNA Stool Mini Kit [Oiagen, Cat. No. 51504]. Lactobacillus spp., Bidobacterium spp., Bacteroides fragilis group, $C$. difficile and $E$. coli quantification was obtained with real-time PCR. Data of microbial counts were subjected to one-way variance analisys in order to evidence significant differences between treated and control group of newborns.

Results Antibiotic therapy reduced the intestinal colonization of Bifidobacterium: $5.51 \mathrm{Log}(\mathrm{CFU} / \mathrm{g})$ in treated samples against 7.07 $\mathrm{Log}(\mathrm{CFU} / \mathrm{g})$ in control samples; $\mathrm{P}<0.05$.

All the others microbial genera and species analysed were not affected by the maternal treatment with Ampicillin.

Conclusions Preliminary results showed a decrease of early Bifidobacterium count in the microbiota of newborns; the clinical meaning or the effect on newborn immunity need to be investigated with larger studies.

\section{EFFICACY OF PROPHYLACTIC FLUCONAZOLE IN REDUCING CANDIDEMIA IN HIGH RISK NICU AND PICU PATIENTS}

doi:10.1136/archdischild-2012-302724.1335

DA Daeem. Pediatrics, Ibn Sina College Hospital, Jeddah, Saudi Arabia

Background Candidal infection is a common cause of morbidity and mortality in neonatal intensive care unit (NICU) and pediatric intensive care unit(PICU) pateints, especially those with risk factors.

Objectives To determine the prevalence of Candida species in risky NICU and PICU patients and evaluate the efficacy of prophylactic Fluconazole in reducing Candida colonization and subsequent invasive candidemia in those patients.

Design Prospective, randomized, double blind placebo controlled clinical study.

Setting Tertiary level intensive care units at pediatric department. Subjects 80 intensive care unit high risk group patient of neonatal and pediatric age.

Intervention Children were randomly grouped during first three days to receive either Fluconazole or placebo till 28 days or less, if discharged or died earlier. Weekly surveillance cultures from oropharyngeal swabs, urine, stool, sputum (when available), and blood.

Results Baseline risk factors for Candida infection in Fluconazole and Placebo groups were similar. Candida colonization was reported in 35 patients (87.5\%) in the placebo group which was significantly higher $(\mathrm{P}=0.0001)$ than that detected among patients in the Fluconazole treated group [10 patients (25\%)]. fluconazole treated group showed significantly lower colonization with Candida albicans (C. albicans) and higher colonization with non Candida albicans (non-C. albicans)versus placebo group. Invasive Candida infection was significantly higher $(\mathrm{P}=0.03)$ among placebo group than Fluconazole treated one. Invasive non-C. albicans infection was reported in $9 / 13$ patients 6 patients $(66.6 \%)$ in Placebo group and 3 patients (33.3\%) in Fluconazole treated group].

Conclusion Prophylactic Fluconazole in risky patients in ICU is effective in reducing Candida colonization but not invasive candidemia.

\section{POSTNATAL SERUM CREATININE TRENDS IN NEONATES: JAFFE COMPARED TO ENZYMATIC QUANTIFICATION TECHNIQUE}

doi:10.1136/archdischild-2012-302724.1336

'A Smits, 'J Kelchtermans, 'S Hendrickx, ${ }^{2} \mathrm{D}$ Mekahli, ${ }^{3} \mathrm{~F}$ Vanstapel, ${ }^{3 \mathrm{P}}$ Vermeersch, ${ }^{2} \mathrm{E}$ Levtchenko, ${ }^{1} \mathrm{~K}$ Allegaert. ${ }^{1}$ Neonatal Intensive Care Unit; ${ }^{2}$ Department of Pediatric Nephrology; ${ }^{3}$ Department of Laboratory Medicine, University Hospitals Leuven, Leuven, Belgium

Background Serum creatinine (Scr) reflects to a certain extent GFR in neonates, but postnatal observations also depends on the technique used (Jaffe colorimetry or enzymatic quantification) as recently quantified in ELBW neonates (1.2). We aimed to assess the impact of enzymatic versus Jaffe quantification and to describe postnatal Scr trends for both techniques in neonates with higher birth weight (3).

Methods Scr values quantified by Jaffe in 1140 neonates were compared to values obtained by enzymatic quantification in 1023 neonates in one NICU. All Scr values collected in the first 42 days of postnatal life were included and postnatal trends for cohorts $<1 \mathrm{~kg}$ 1-2 kg, 2-3 kg and $>3 \mathrm{~kg}$ were compared.

Results Postnatal patterns were similar between both techniques, with an initial increase of Scr (highest and last in the smallest neonates) in early postnatal life, and a subsequent decrease, most 
delayed in the smallest neonates. For all consecutive postnatal observations, Jaffe always resulted in higher Scr compared to the enzymatic technique, but the differences in median values between both techniques $(0.1-0.26 \mathrm{mg} / \mathrm{dl}$, equal to $8.8-23 \mu \mathrm{mol} / \mathrm{l})$, were not a fixed value.

Conclusions When using Scr to estimate renal function in neonates, clinicians should in addition to postnatal changes and other covariates of renal function, also consider the technique applied. There is no fixed conversion factor to correct for differences between both techniques.

1. Allegaert, J Matern Fetal Neonatal Med DOI 10.3109/14767058.2012.657277.

2. Kuppens, J Matern Fetal Neonatal Med DOI 10.3109/14767058.2011.602144.

3. Bueva, Pediatr Res 1994

\section{NEWBORN AND ADULT MONOCYTES SHOW DIFFERENT INFLAMMATORY RESPONSES TO BACTERIAL INFECTION: POTENTIAL ROLE OF MAPK INHIBITION}

doi:10.1136/archdischild-2012-302724.1337

EM Speer, AJ Chander. Pediatrics, Stony Brook University, Stony Brook, NY, USA

Background and Aims The neonatal inflammatory response is associated with adverse outcomes like chronic lung disease. Recent studies suggested that newborn innate immune responses differ from adults. We aim to compare the expression of inflammatory cytokines between newborn and adult monocytes, and to investigate the mechanisms underlying the differential response.

Methods Purified monocytes from 15 healthy term newborns (C-section, no labor or chorioamnionitis) and 15 healthy adults (no infection), were cultured (90min) and stimulated without or with $0.1 \mathrm{ng} / \mathrm{ml}$ or $10 \mathrm{ng} / \mathrm{ml}$ LPS for 4 or 24 hours. Cells were harvested and RNA extracted. mRNA expression was determined with real-time PCR and normalized to $\beta 2$-microglobulin as housekeeping gene. Results were analyzed by ANOVA and Students t-test with $p \leq 0.05$ considered significant.

Results Results are discussed in comparison to control values. Newborn monocytes showed increased IL6 $(0.1 \mathrm{ng} / \mathrm{ml}$ or $10 \mathrm{ng} / \mathrm{ml}$ LPS) and TNF $\alpha(0.1 \mathrm{ng} / \mathrm{ml}$ LPS) expression after $4 \mathrm{~h}$, whereas IL10mRNA was lower after $4 \mathrm{~h}$ and $24 \mathrm{~h}$ LPS compared to adults. LPS-stimulation increased NFKBp65 expression in adults but not in newborns at $24 \mathrm{~h}$. IKB $\alpha$ and Toll-interacting protein were comparable between groups. IRAK3 (TLR4-pathway regulator) was elevated in newborns at 4 and $24 \mathrm{~h}$ with LPS-stimulation, but only at $24 \mathrm{~h}$ in adults. Dual specificity phosphatase 1 (DUSP1) was significantly lower in newborn monocytes compared to adults after 24h LPS at both concentrations.

Conclusions Newborn compared to adult monocytes show increased expression of inflammatory cytokines. Diminished upregulation of DUSP1 (negative regulator of MAPK-pathway) might explain the enhanced pro-inflammatory profile of newborn compared to adult monocytes after microbial stimulation.

\section{HIGH PROPORTION OF INTESTINAL ESBL COLONIZATION AMONG INFANTS AT A NEONATAL INTENSIVE CARE UNIT IN A TERTIARY HOSPITAL IN ECUADOR}

doi:10.1136/archdischild-2012-302724.1338

IV Nordberg, ${ }^{2} \mathrm{~A}$ Quizphe, ${ }^{3} \mathrm{C}$ Giske, ${ }^{3} \mathrm{~A}$ Iversen, ${ }^{2} \mathrm{~T}$ Galindo, ${ }^{2} \mathrm{E}$ Ochoa, 'L Navér. ${ }^{1}$ Department of Clinical Science, Intervention and Technology (CLINTEC)/Division of Paediatrics, Karolinska Institute, Karolinska University Hospital, Stockholm, Sweden; 2Faculty of Medical Science, University of Cuenca. Hospital Vicente Corral Moscoso, Cuenca, Ecuador; ${ }^{3}$ Department of Microbiology, Tumor and Cell Biology (MTC), Karolinska University Hospital, Stockholm, Sweden

Background and Aims Neonatal infections caused by Extendedspectrum beta-lactamase (ESBL)-producing bacteria are associated with increased morbidity and mortality. No data are available on neonatal colonization with ESBL-producing bacteria in Ecuador. The study aimed to assess the proportion of intestinal colonization with ESBL-producing Enterobacteriacae and their resistance pattern among infants hospitalized at the neonatal intensive care unit, Cuenca, Ecuador.

Methods From February to April 2011, stool specimens were collected, every two weeks, from all hospitalized neonates. Rectal swabs were plated on Mac Conkey agar containing cefotaxime and ceftazidime. Species identification and susceptibility tests were confirmed with Vitek2 and the epidemiologic typing was performed using Diversilab (Both bioMérieux).

Results 137 specimens were collected from 78 patients and $61.5 \%$ of the neonates became colonized with ESBL. The majority of the strains were Escherichia coli (EC, 88.5\%) followed by Klebsiella pneumoniae (KP, 11.5\%). Gentamicin resistance occurred in $98.6 \%$ of the EC and $100 \%$ of the KP and ciprofloxacin resistance in $98.6 \%$ of the $\mathrm{EC}$ and $0 \%$ of the KP strains. All strains were susceptible to carbapenems. Epidemiologic typing divided the EC isolates in two clusters and one unique isolate and the KP isolates were divided in two clusters. All EC and KP had bla $a_{\text {СтХ-м }}$ group 1 except for the unique EC isolate that had bla $a_{\text {CTX-M }}$ group 9.

Conclusions The high proportion of patients colonized with four clones of ESBL-producing bacteria, reinforces the necessity for implementing surveillance programs as well as improved infection control to prevent further spread of ESBL strains between hospitalized neonates.

\section{THE VALUE OF PLACENTAL PATHOLOGICAL AND MICROBIOLOGICAL ASPECTS ON PRETERM DELIVERY AND OUTCOME}

doi:10.1136/archdischild-2012-302724.1339

${ }^{1}$ A Korraa, ${ }^{2} Z$ Nabil, ${ }^{3} \mathrm{~N}$ Samir, ${ }^{1} E$ El Geriany. ${ }^{1}$ Pediatrics; ${ }^{2}$ Microbiology, Faculty of Medicine for Girls, Al Azhar University; ${ }^{3}$ Pathology, Ain Shams University, Cairo, Egypt

Background Both clinical findings and the high incidence of decidual inflammation/infection in placentasare associated with preterm deliveries.

Objectives To find out the relation of histopathology and microbiology findings of the placenta and preterm birth and to document the association of placental changes and neonatal outcome.

Methods and results A comparative, analytical study was carried out on Placentas from 100 mothers, 50 with preterm delivery (case group), and 50 with full term delivery(control group). Pathology of the placentas and PCR to detect bacterial SrDNA were performed for the placentae and neonates. Preterm Placentas showed a significantly higher inflammatory lesions than those of full term placentas, (68\% in preterm versus $4 \%$ in full term). The percentage of bacterial isolation by PCR from preterm placenta was significantly higher than full term placenta ( $75 \%$ vs $22 \%$ ), suggesting that most of unexplained preterm delivery is inflammation and/or infection related. The study demonstrated significant association between placental and neonatal bacterialSrDNA. Our results showed that placental inflammatory lesions were significantly associated with lower gestational age, lower weight and length of preterm neonates. On follow up of the preterm neonates, the percentage of RDS, SGA, BPD and neonatal mortality rate were higher among preterm with placental inflammation/infection than those without.

Conclusion Infection of the placenta is associated strongly with histological chorioamnionitis and preterm birth Placental pathology is very useful in identifying undiagnosed subclinical maternal infection. The percentage of neonatal morbidity and neonatal mortality were higher in cases with positive placental findings for inflammation and infection. 